\title{
A Computer Assisted Planning System for the Placement of sEEG Electrodes in the Treatment of Epilepsy
}

\author{
G Zombori ${ }^{1}$, R Rodionov ${ }^{2,3}$, M Nowell ${ }^{2,3}$, MA Zuluaga ${ }^{1}$, M Clarkson ${ }^{1}$, C Micallef \\ 2,3 B Diehl $^{2,3}$, T Wehner ${ }^{2,3}$, A Miserochi ${ }^{2,3}$, A McEvoy ${ }^{2,3}$, J Duncan ${ }^{2,3}$, S Ourselin ${ }^{1,4}$ \\ 1. Centre for Medical Image Computing, University College London, UK \\ 2. Dept. of Clinical and Experimental Epilepsy, UCL Institute of Neurology, London, UK \\ 3. National Hospital for Neurology and Neurosurgery (NHNN), London, UK \\ 4. Dementia Research Centre, Department of Neurodegenerative Disease, UCL Institute of \\ Neurology, London, UK
}

\begin{abstract}
Approximately $20-30 \%$ of patients with focal epilepsy are medically refractory and may be candidates for curative surgery. Stereo EEG is the placement of multiple depth electrodes into the brain to record seizure activity and precisely identify the area to be resected. The two important criteria for electrode implantation are accurate navigation to the target area, and avoidance of critical structures such as blood vessels. In current practice neurosurgeons have no assistance in the planning of the electrode trajectories.

To provide assistance a real-time solution was developed that first identifies the potential entry points by analysing the entry-angle, then computes the associated risks for trajectories starting from these locations. The entry angle, the total length of the trajectory and distances to critical structures are presented in an interactive way that is integrated with standard electrode placement planning tools and advanced visualisation. We show that this improves the planning of intracranial implantation, with safer trajectories in less time.
\end{abstract}

\section{Introduction}

Approximately $20-30 \%$ of patients with focal epilepsy are medically refractory to treatment with anti-epileptic drugs. These patients are potential candidates for curative respective surgery [1]. The primary aim of epilepsy surgery is to remove the epileptogenic zone-'the minimum amount of cortex that must be resected (inactivated or completely disconnected) to produce seizure freedom' [2]. The identification of the epileptogenic zone often requires the placement of intracranial electrodes to record where seizures start and rapidly propagate. Stereo-electroencephalography (SEEG) is the practice of recording electroencephalographic signals via depth electrodes that are surgically implanted into the brain tissue. The challenge in epilepsy surgery now is in the treatment of the more difficult patient groups (extratemporal non-lesional) where SEEG is increasingly utilised. This invasive investigation carries the risks of infection, haemorrhage and neurological deficit [3]. In the current work we only consider SEEG electrode implantation where brain shift is anticipated to be negligible due to the borehole surgery approach.

adfa, p. 1, 2011.

(C) Springer-Verlag Berlin Heidelberg 2011 
Preoperative planning of SEEG electrode placement is a necessary prerequisite to implantation. Important anatomical and functional landmarks of the brain (such as blood vessels, pial boundaries, nerve tracts, etc.) can be identified with advanced neuro-imaging and image-processing techniques. SEEG electrode trajectories are defined by a target area that has to be reached by the electrode and an entry point where the electrode penetrates the skull. Electrode arrangements are planned to achieve adequate cortical coverage and pass through safe, avascular planes. The large number of electrodes required in SEEG and the cumulative risk associated with this implies that assisted planning (AP) is the most useful in these clinical cases.

Previous publications on pre-operative planning of depth electrode placement describe approaches to find the optimal path either automatically [4-6] or by assisting the decision making process of the neurosurgeon [7-9]. Another state of the art approach [10] proposed a system to assist planning at all stages of the planning from the selection of the target point to the selection of a safe entry point that minimizes the risk of hitting with vital structures. In all of these approaches the operator needs to select the target point precisely and the time required to compute the optimised paths is generally long. A recent article describes a high performance solution to enable quantitative estimation of the risk associated with a particular access path at interactive rates. The authors employ Graphics Processing Units (GPUs) to achieve real-time speed and use risk maps visualisation to aid the planning process [11].

Here we present an advanced set of tools for computer-assisted planning of SEEG electrode placement that come as part of our surgical planning system EpiNav ${ }^{\mathrm{TM}}$ (CMIC, UCL, London, UK) that allows neurosurgeons to define safer trajectories in less time. EpiNav ${ }^{\mathrm{TM}}$ advances on previous work by offering improved real-time visual feedback to planning; including the addition of several vital structures (such as functional brain regions) into the risk assessment and also factoring the entry angle of the trajectory line with the skull surface.

\section{Methods}

To enable a faster planning process and ensure safety of the resulting implantation plan the following conditions have to be met with the aid of the planning system:

1. Critical structures have to be clearly identifiable (visualisation).

2. None of the trajectories can intersect any critical tissue to avoid harm to the patient.

3. The trajectory should be further from any critical tissue by a specified safety margin based on the accuracy of the surgical procedure of implanting the electrodes.

4. The trajectory should be as short as possible. It is assumed here that only the tip of the electrode is meant to hit the target. At this stage we are not considering cases of multiple targets sampled by one electrode.

5. The entry angle of the trajectory should be as close to 90 degrees as possible to allow robust implementation of the planned entry angle during the surgical procedure. 
To achieve fast processing and real-time interaction EpiNav ${ }^{\mathrm{TM}}$ was developed using a cross platform C++ library NifTK (www.niftk.org) that is based on the Medical Imaging and Interaction Toolkit (MITK, www.mitk.org). Furthermore we employ a modern graphics card (GPU) and utilize the OpenCL library to enable parallel programming. EpiNav ${ }^{\mathrm{TM}}$ can be installed on any recent PC that runs Mac OS X, Linux or Windows and has a GPU that is OpenCL 1.1 compatible.

\subsection{Critical structures}

Identifying critical objects is key to successful estimation of a safe trajectory. The critical structures are imported into an interactive visualisation workstation using the functionality of EpiNav ${ }^{\mathrm{TM}}$, then converted into 3D surface mesh objects and coloured using a colour scheme (See Fig. 3/a) as in our previous work [12]. The clinically relevant landmarks are white matter tracts (e.g. cortico-spinal tract, optic radiation tract) derived from DTI data, lesions, eloquent cortex (e.g. language or motor areas) derived from fMRI, areas of ictal hyperperfusion derived from SPECT, areas of hypometabolism derived from PET image, ictal or interictal EEG/MEG sources. Blood vessel images were acquired using CTA, 3D Phase Contrast MR imaging and in some cases ToF MR, then the vasculature was extracted using a custom tool. A surface representation of the skull is used to determine the accurate location of the entry points and to compute the entry angle of the trajectory. The skull surface is usually derived from CT, CTA or pseudo CT synthetized from an MR scan.

\subsection{Trajectory Planning}

The planning process starts with the selection of the target point. The target point can be placed by clicking on any location within the space of the reference image on one of the $2 \mathrm{D}$ or $3 \mathrm{D}$ views. If a detailed brain parcellation map is available it can be used to aid the target selection, by highlighting various anatomical regions of the brain. Entry points can only be placed onto the skull surface. In manual mode this is ensured by the entry point selection tool.

\subsection{Entry Points Search and Risk Analysis}

As soon as the target point is selected the system will analyse the topology of the critical structures and offer a set of entry points that represent minimal risk. The entry point search algorithm is a fully automatic method that is implemented on the GPU. It takes the skull mesh as the input and processes each of its vertices, so the sampling rate is defined by the number of vertices in the skull model. The algorithm takes into account the distance of the target point and the currently evaluated vertex (i.e. the length of the trajectory) and the entry angle of the trajectory starting from this vertex. From a surgical point of view the entry angle has to be as close to perpendicular as possible, otherwise it is not possible to drill the borehole through the skull. As the angle and length analysis is computationally inexpensive it is practical to perform it as the first step and disqualify entry points that are too far, or the entry angle is outside a 
user-configured range (in our practice \pm 10 degrees from perpendicular). The remaining entry point candidates are checked for collisions with the critical structures: the ones that do not allow straight access are ruled out. Early exclusion of unsuitable entry points avoids unnecessary evaluations, which enables more detailed risk analysis on the remaining points while maintaining real time performance.

The associated risk to each potential trajectory is evaluated using a GPU based module. Previously published risk metrics would assign risk based on the shortest Euclidean distance from any point on the trajectory, while our new metric provides a full distance profile along it. For each potential trajectory 256 sample locations are considered along the length from entry point to target point. The minimum distance to the critical structures (one at a time) is computed per sample point and the results are aggregated, resulting in an array of values that contains the distance to the nearest critical object per sample point.

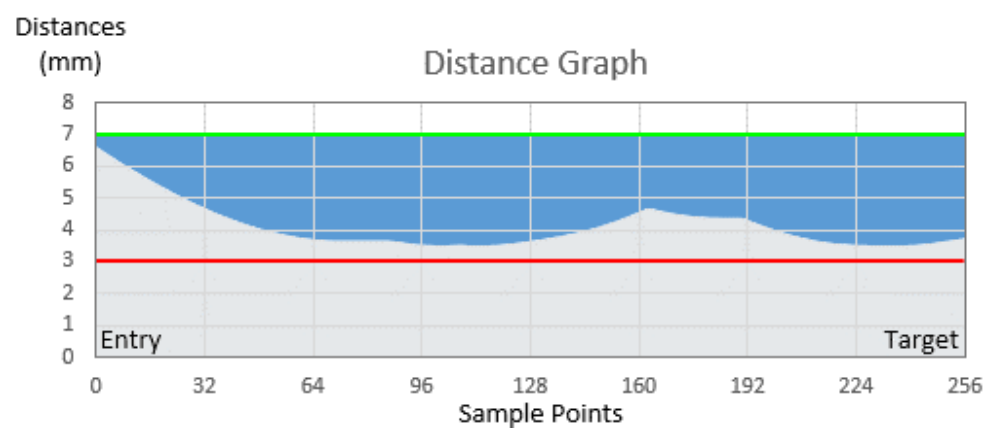

Fig. 1. Distance Graph. The light grey area represents the distance to a critical structure. Green Line - Risk Zone threshold; Red line - Safety margin; Grey/Blue border line - Distance Graph with blue area reflecting risk and grey area reflecting minimal distance to the nearest object.

Once the critical distances have been identified for each potential trajectory the actual risk computation can take place. In this work a new integrative risk metric is introduced that quantifies the level of risk on a $0-1$ scale $(0-$ no risk; 1 - the highest risk which must be avoided). The proposed metric can be extended to include distance, angle of entry and whether the electrode contacts are in grey matter.

A trajectory is considered too risky when the minimum distance to any critical structure is smaller than the "Safety Margin" distance $d_{\min }$. If the trajectory lies further from any structure than a "Risk Zone" margin $d_{\max }$ then it represents no potential harm. In EpiNav ${ }^{\mathrm{TM}}$ these values can be adjusted by the user when required. The amount of risk that emanates from proximity to critical structures $f_{\text {dist }}$ can be quantified within a range of accepted values by computing the area above the curve (See Fig. 1 - blue area):

$$
S_{\text {risk }}=\int_{\text {entry }}^{\text {target }}\left(d_{\text {max }}-\left(f_{\text {dist }}(x)-\mathrm{d}_{\text {min }}\right)\right) d x
$$




$$
R_{\text {dist }}=\frac{S_{\text {risk }}}{\left(d_{\max }-d_{\min }\right) * \text { length }}
$$

This formula yields risk values in the range of $0-1$. The quality of the entry angle can be similarly evaluated $R_{\text {angle }}$ given the range of accepted values, as well as the length of the trajectory $R_{\text {length }}$. These independent risk components can be combined by applying certain weight factors:

$$
\begin{gathered}
R_{\text {total }}=w_{1} R_{\text {dist }}+w_{2} R_{\text {angle }}+w_{3} R_{\text {length }}, \\
\text { where } \sum w_{i}=1 \text { and } R_{i} \in[0 \sim 1]
\end{gathered}
$$

This final metric $R_{\text {total }}$ describes the overall quality of the trajectory. After every potential trajectory has been assessed the risk values are visualised in form of a riskmap (Fig. 2.). The planning module will automatically suggest to use the trajectory that has the lowest risk value across the whole map. However, the surgeon can override this by modifying the entry point according to the risk map.

The technical challenges of this work are related to the efficient parallel implementation of collision detection, proximity search and distance evaluations in OpenCL. To allow real-time performance a Bounding Volume Hierarchy $(\mathrm{BVH})$ is built over the cells of critical structures, that is an acceleration data structure used to facilitate the fast traversal of large datasets containing 3D points. Discussion of the specific implementation details will be presented in our future publication, however a good description of the use of BVH for proximity analysis using GPU hardware can be found here $[11,13,14]$.

\section{$2.4 \quad$ Visualisation}

EpiNav ${ }^{\mathrm{TM}}$ provides the standard ortho-view (2D planes: axial, coronal, sagittal), combined with 3D visualisation (volume / surface rendering) in a $2 \times 2$ layout. The "Probe Eye View" display and a "Distance Graph" widget are placed in a separate window. The Probe Eye View displays an oblique plane (2D) that is always perpendicular to the line of the trajectory (See Fig. 3/b). The distance graph widget (Fig. 3/c) provides the visual representation of the minimal distance information in form of a graph. The length of the graph (horizontal axis) corresponds to the length of the trajectory, while the height of the bars (vertical axis) represent the distance to the nearest critical structure for that particular point of the trajectory. The graph is re-scaled along the vertical axis to focus the representation on the critical sections (Risk Zone).

EpiNav $^{\mathrm{TM}}$ offers linked visualisation components (similarly to [10]) where the cursor location is synchronised between all visualisation components. For example picking a surface point in the 3D window will update the position of all other views. Similarly, clicking on a point of the distance graph will update the slice positions in the 3 orthogonal plane views and will also update the displayed slice in the probe eye view. This behaviour allows the user to visually identify risky sections of the planned trajectory, by clicking on these sections on the risk display the associated 2D and 3D views will be presented for review. 


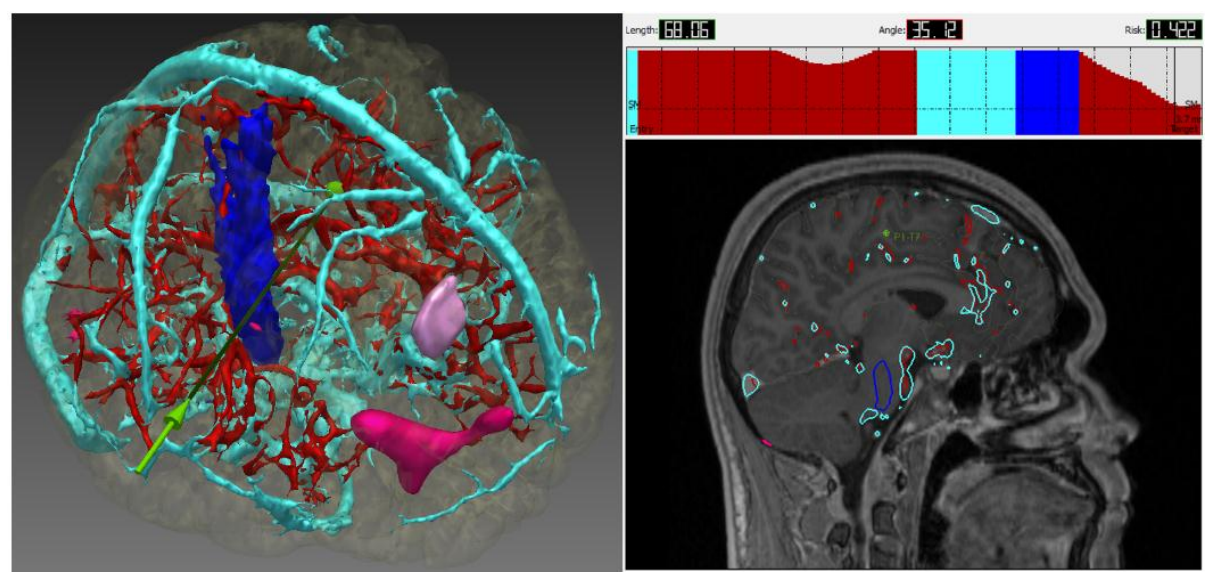

Fig. 3/a: Visualisation of various critical structures. Blue: Cerebrospinal tract (CST), Pink: hypometabolism PET, Deep Pink: SPECT, Cyan-Red: blood vessels, Green arrow: Historical trajectory $\mathbf{3 / b}$ : Probe Eye View displaying a projection that is perpendicular to the trajectory with distance graph showing distances to critical structures along the trajectory. Above the graph the length of the path, angle of entry and the risk are displayed.

The locations of the suitable entry points are marked on the skull surface by colouring their location accordingly (Fig. 4). The risk values are linearly mapped onto a colour lookup table that extends from red to green, red meaning high risk while green symbolising low-risk. All information in the risk map, distance graph, as well as the angle, risk, length and slice position are updated real time as the trajectory being adjusted.

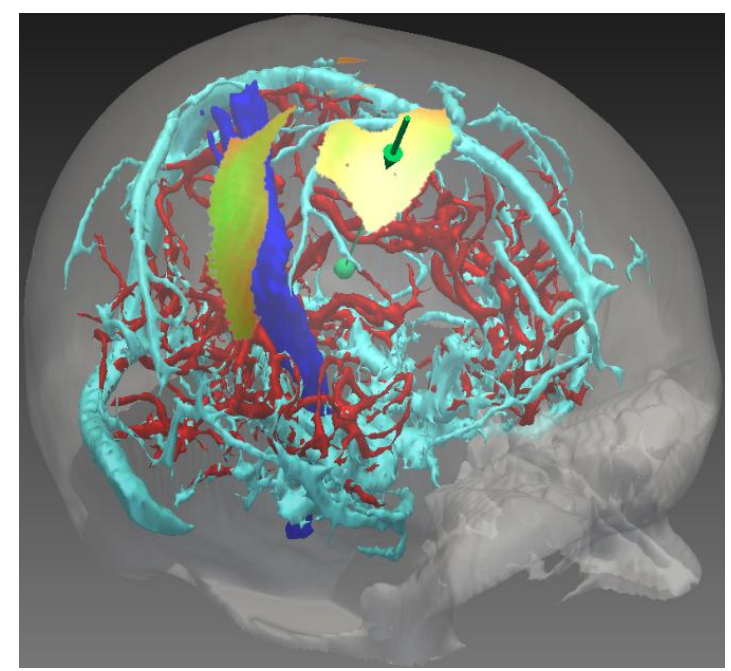

Fig. 4. Risk Map visualisation: The semi-transparent part of the skull represent non-suitable points while the coloured patch shows the potential entry points with associated risk. Green low risk, red - high risk. In this case the target point was picked at random. 


\section{$3 \quad$ Evaluation and results}

\subsection{Computational Performance}

To evaluate the efficiency each of the proposed methods were tested several times using different input data and the average execution time was recorded for each case. In the evaluation 4 surface meshes were used: skull surface (185k vertices); cerebrospinal tract (33k vertices); veins (91k vertices); arteries (70k vertices). The process times were recorded using OpenCL time events, including both the execution time of the OpenCL kernels, task executions scheduling and the time of data transfer between host and device. The desktop computer that was used in the tests has the following configuration: Intel XEON 16core CPU, 16 GB of RAM and an NVidia Quadro K2000 2 GB GPU.

The first step of the risk analysis is the entry point search algorithm that aims to reduce the number of trajectory candidates. To test this the skull surface model was loaded and 10 historical electrode target points were selected. The average time to complete was measured to be $2.7 \mathrm{~ms}$, while the average reduction factor was $97 \%$, resulting between 200-6000 entry point candidates. The next step is the construction of the BVH that only needs to be constructed once at the start. For the reference skull image (the largest mesh used in the test) the construction took $50 \mathrm{~ms}$ on average.

\begin{tabular}{|c|c|}
\hline Total Num. of Trajectories & Risk Evaluation (ms) \\
\hline 1 & 1.3 \\
\hline 5 & 7.1 \\
\hline 50 & 13.4 \\
\hline 500 & 96.7 \\
\hline 5000 & 248.1 \\
\hline
\end{tabular}

Table 1. Computation time of risk evaluation for software generated trajectories.

To evaluate risk computation times various number of trajectories were generated by specifying a target point and assigning a number of random entry points. The computation time was found to be a linear function of the number of trajectory candidates, for the expected maximal number of entry points ( 6000) the AP module provides close to real time interactions with 4 fps (Table 1 ).

\subsection{Neurosurgical evaluations}

To evaluate the benefits of employing the new risk estimation, the risk map and distance graph, 30 electrode trajectories in 6 patients were evaluated, and their length (Fig. 5), angle of entry (Fig. 6) risk value (Fig. 7) were compared to results of nonassisted planning. The analysis was performed using historical data, where the original electrode trajectories were planned by expert neurosurgeons who relied only on traditional 2D visualisation. The average time to plan one electrode using the nonassisted approach is estimated as $10-15$ min based on our previous experience. The new trajectories were planned by a neurosurgeon using the computer assisted plan- 
ning (AP), keeping the same target points for the purpose of comparison.

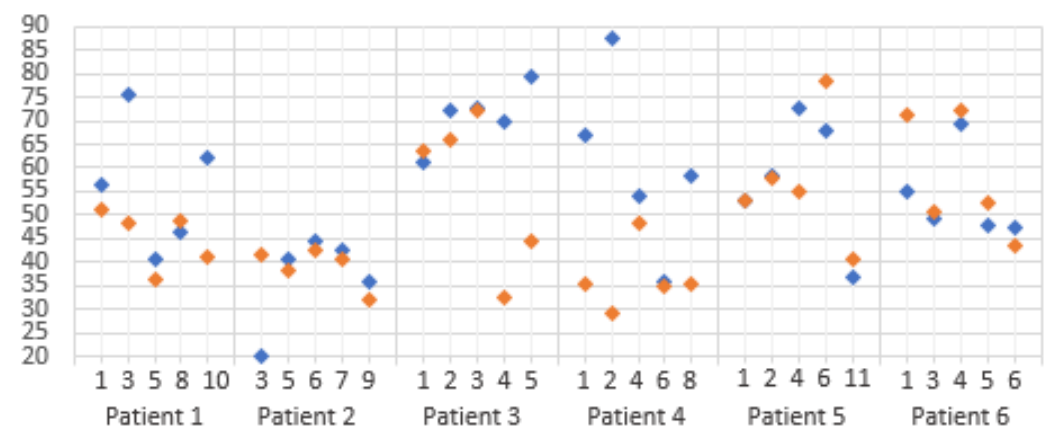

Fig. 5. Comparison of length. Values in blue represent trajectories that were planned without $\mathrm{AP}$, while results in orange were acquired using the AP module.

For each target point, the AP module analysed the topology of the critical structures to find potential entry points and computed the risks for them. Based on these risk values the system automatically offered an optimal entry for the new trajectory.

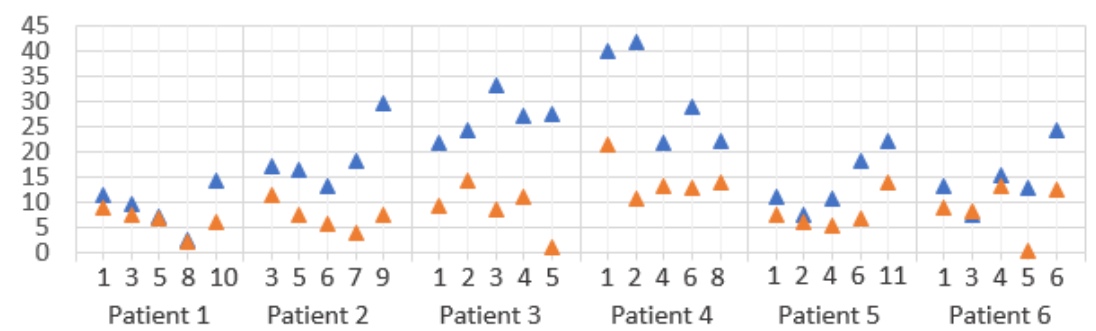

Fig. 6. Comparison of entry angle. Values in blue represent trajectories that were planned without $\mathrm{AP}$, while results in orange were acquired using the AP module.

The new trajectories were inspected by the surgeon to validate the safety profile and feasibility. The entry point has changed in all cases (Fig. 8), while the target point had to be adjusted in three cases (P2-T5, P3-T4, P5-T4) when it was placed too close to a critical structure originally (without assistance - Fig. 3/b), which made AP impossible.

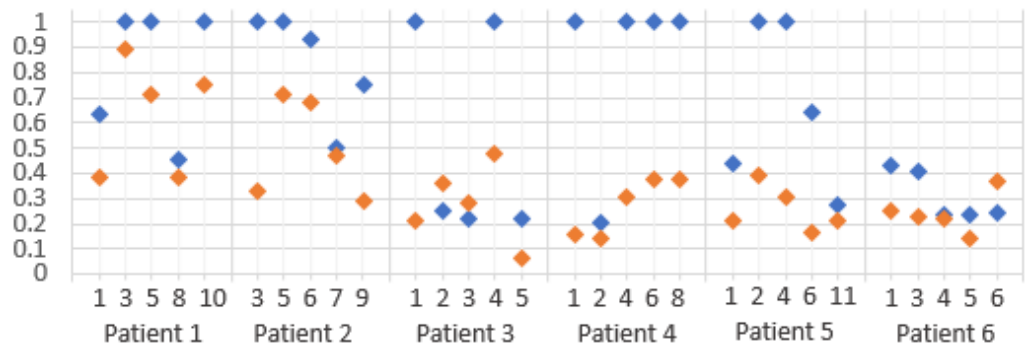

Fig. 7. Comparison of risk. Values in blue represent trajectories that were planned without the $\mathrm{AP}$, while results in orange were acquired using the AP module. 
The AP module provided a more feasible angle in all cases, while length of the trajectory was shorter in 57 of the 60 cases. The overall risk was smaller in 57 of the 60 cases using the AP, for the remaining 3 cases the risk was only marginally higher while both angle and length values were better. The general feedback from the surgeon was that the new system provides trajectories that are easier to implement in theatre and a lower risk profile by locating feasible entry points. The required planning time reduced to $2-3$ min per electrode, which is approximately the time it takes to thoroughly inspect the full length of the planned trajectory.

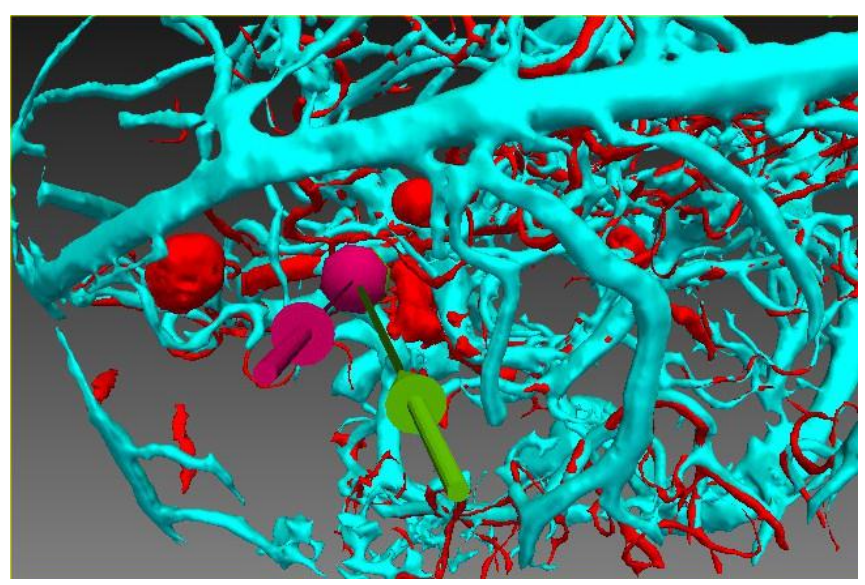

Fig. 8. Change in the Trajectory. Green: Old path; Purple: New path from assisted planning. The increased distance to blood vessels can be observed.

\section{Conclusion}

We have demonstrated that EpiNav ${ }^{\mathrm{TM}}$ finds safer trajectories that are easier to implement and gives the surgeon greater confidence in individual electrode trajectory. The GPU based implementation enables real-time interaction and risk evaluation that reduces planning time and allows a more efficient clinical workflow. One limitation with assisted planning is the reliance on the quality of segmented surfaces that are used. As new imaging and segmentation tools become available, assisted planning will become increasingly reliable. Future work will concentrate on optimising electrode efficiency by segmenting out grey and white matter, and by simulating electrode contacts. The user will then be able to combine safety, feasibility and efficiency scores to select the most appropriate trajectory. The next logical advance on assisted planning is to incorporate electrode arrangements instead of individual electrodes and to add a semi-automatic target placement by employing anatomical parcellation tools.

Acknowledgements: This publication presents independent research supported by the Health Innovation Challenge Fund (HICF-T4-275), a parallel funding partnership between the Department of Health and Wellcome Trust. The views expressed in this publication are those of the author(s) and not necessarily those of the Department of 
Health or Wellcome Trust.

\section{$5 \quad$ References}

1. Sirven, J.I., Pedley, T.A., Wilterdink, J.L.: Evaluation and management of drug-resistant epilepsy. (2011) Available at: http://www.uptodate.com/contents/evaluation-andmanagement-of-drugresistant-epilepsy. Accessed Nov 222013

2. David, O., Blauwblomme, T., Job, A.-S., Chabardès, S., Hoffmann, D., Minotti, L., Kahane, P.: Imaging the seizure onset zone with stereo-electroencephalography. Brain 134, 2898-2911 (2011)

3. Olivier, A., Boling, W.W., Tanriverdi, T.: Techniques in epilepsy surgery: the MNI approach. Cambridge University Press (2012)

4. Bériault, S., Al Subaie, F., Mok, K., Sadikot, A.F., Pike, G.B.: Automatic trajectory planning of DBS neurosurgery from multi-modal MRI datasets. Medical Image Computing and Computer-Assisted Intervention-MICCAI 2011, pp. 259-266. Springer (2011)

5. Essert, C., Haegelen, C., Lalys, F., Abadie, A., Jannin, P.: Automatic computation of electrode trajectories for Deep Brain Stimulation: a hybrid symbolic and numerical approach. International journal of computer assisted radiology and surgery 7, 517-532 (2012)

6. De Momi, E., Caborni, C., Cardinale, F., Castana, L., Casaceli, G., Cossu, M., Antiga, L., Ferrigno, G.: Automatic Trajectory Planner for StereoElectroEncephaloGraphy Procedures: A Retrospective Study. Biomedical Engineering, IEEE Transactions on 60, 986-993 (2013)

7. Navkar, N.V., Tsekos, N.V., Stafford, J.R., Weinberg, J.S., Deng, Z.: Visualization and planning of neurosurgical interventions with straight access. Information Processing in Computer-Assisted Interventions, pp. 1-11. Springer (2010)

8. Shamir, R.R., Tamir, I., Dabool, E., Joskowicz, L., Shoshan, Y.: A method for planning safe trajectories in image-guided keyhole neurosurgery. Medical Image Computing and Computer-Assisted Intervention-MICCAI 2010, pp. 457-464. Springer (2010)

9. Bériault, S., Al Subaie, F., Collins, D.L., Sadikot, A.F., Pike, G.B.: A multi-modal approach to computer-assisted deep brain stimulation trajectory planning. International journal of computer assisted radiology and surgery 7, 687-704 (2012)

10. Herghelegiu, P.-C., Manta, V., Perin, R., Bruckner, S., Gröller, E.: Biopsy Planner-Visual Analysis for Needle Pathway Planning in Deep Seated Brain Tumor Biopsy. In: Computer Graphics Forum, pp. 1085-1094. Wiley Online Library, (2012)

11. Rincon, M., Navkar, N., Tsekos, N., Deng, Z.: GPU-Accelerated Interactive Visualization and Planning of Neurosurgical Interventions. (2013)

12. Rodionov, R., Vollmar, C., Nowell, M., Miserocchi, A., Wehner, T., Micallef, C., Zombori, G., Ourselin, S., Diehl, B., McEvoy, A.W.: Feasibility of multimodal 3D neuroimaging to guide implantation of intracranial EEG electrodes. Epilepsy research 107, 91-100 (2013)

13. Lauterbach, C., Garland, M., Sengupta, S., Luebke, D., Manocha, D.: Fast BVH construction on GPUs. In: Computer Graphics Forum, pp. 375-384. Wiley Online Library, (2009)

14. Karras, T.: Maximizing parallelism in the construction of BVHs, octrees, and k-d trees. In: Proceedings of the Fourth ACM SIGGRAPH/Eurographics conference on HighPerformance Graphics, pp. 33-37. Eurographics Association, (2012) 